\title{
Plac1 Expression Pattern at the Mouse Fetomaternal Interface and Involvement in Trophoblast Differentiation
}

\author{
Yanli Gu ${ }^{a} \quad$ Junhui Wan ${ }^{b} \quad$ Lv Yao $^{a} \quad$ Nan-Ni Peng ${ }^{c}$ Wen-Lin Changa,d \\ aGuangdong and Shenzhen Key Laboratory of Male Reproductive Medicine and Genetics, Institute of \\ Urology, Peking University Shenzhen Hospital, Biomedical Research Institute, Shenzhen Peking University- \\ The Hong Kong University of Science and Technology Medical Center, Guangdong, 'Department of \\ Gynaecology and Obstetrics, The First Affiliated Hospital of Nanchang University, Nanchang, Jiangxi, \\ 'Reproductive Medical Center of The $3^{\text {rd }}$ Affiliated Hospital of Shenzen University, Shenzhen, Guangdong, \\ ${ }^{d}$ College of Veterinary Science, Hunan Agricultural University, Changsha, Hunan, China
}

\section{Key Words}

Plac1 - Spatiotemporal expression pattern - Fetomaternal interface - Trophoblast • Differentiation $\cdot$ Placenta

\begin{abstract}
Background/Aims: It is well known that Plac1 is a placenta-specific gene; however, its spatiotemporal expression pattern and exact role at the mouse fetomaternal interface re $\mathrm{m}$ a in s unclear. Methods: In situ hybridization (ISH) was used to localize the Plac1 mRNA at the mouse fetomaternal interface. A trophoblast stem cell (TS) differentiation model with Plac1 shRNA-overexpressing lentivirus was employed to investigate the possible role of Plac1 in placentation. Real-time RT-PCR was used to detect changes in gene expression. Results: Plac1 was exclusively expressed in the ectoplacental cone (EPC) as well as in 8.5 and 9.5 days post-coitum (dpc) embryos. Subsequently, Plac1 expression was abundant in the spongiotrophoblast layer and moderately in the labyrinth layer until $13.5 \mathrm{dpc}$, and declined thereafter. Interestingly, Plac1 was also expressed by secondary trophoblast giant cells and glycogen trophoblast cells, but not in primary trophoblast giant cells. Plac1 transcription was increased during the TS differentiation $(P<0.01)$, and knockdown of Plac1 significantly impaired TS differentiation. Conclusion: Plac1 is abundantly expressed at the fetomaternal interface and in all trophoblast subtypes except in primary trophoblast giant cells. Plac1 knockdown retarded the progress of TS differentiation, indicating that Plac1 is necessary for normal trophoblast differentiation into various trophoblast subpopulations.
\end{abstract}

\section{Introduction}

(C) 2017 The Author(s)

Published by S. Karger AG, Basel

The placenta is a transient mammal-specific organ that controls the exchange of substance between the mother and fetus. Placental mammals originated about 100 million years

Wen-Lin Chang

and Nan-ni Peng

\section{KARGER}


Gu et al.: Plac1 Expression Pattern and Function in Mouse Placentas

ago, and the placenta ensured a successful pregnancy [1]. During the long-term enveloped process, the placenta has evolved with high diversity both in structure and function across different mammalian species. Primates and rodents showed the least evolutionary distance in terms of placental structure, and have a typical hemochorial placenta, which characterizes the most intimate fetomaternal contact [2]. Thus, mouse and rat models are often used to study placentas as the findings often help understand what happens in human placentas.

The complex diversity of mammalian placentas is determined by their unique regulatory mechanisms of specific genes, which typically have spatiotemporal expression patterns during normal placentation [3-6]. Many studies have investigated these genetic mechanisms, including epigenetic regulation within the placenta, regulatory noncoding RNAs, and classical protein-coding genes, all of which contribute to the structural and functional specificity of the placenta [7-10]. While studies have investigated and provided extensive insights into hemochorial placenta development [4], gaps in knowledge remain, and the precise blueprint of genetic regulating mechanisms for normal placentation remains to be fully understood.

Although Rawn [4] summarized that enveloping the placenta did not involve an entirely new set of genes, the mammalian genome has evolved in concert with placental development and there are some genes that are unique to the placenta alone. A series of genes that were initially reported to be enriched in a placental cDNA library are termed placenta-specific genes (Placs). Of these, some genes such as Plac1 have undergone functional studies and have subsequently been established in mouse models [11]. Plac1-mutated mice have been shown to have abnormal placental structure and the viability of postnatal offspring of these mutant mice have been found to be significantly affected. In fact, the precise expression pattern of Plac1 is still not fully clear; this poses a barrier in fully understanding about how Plac1 ensures normal placentation. Thus, this study aims to reveal the exact spatiotemporal expression pattern of Plac1 at the fetomaternal interface in mice. Furthermore, the exact role of Plac1 in trophoblast differentiation will be investigated.

\section{Materials and Methods}

\section{Animals}

In this experiment, 10-week-old CD-1 mice were mated and the presence of a vaginal plug the following morning was used to determine the exact developmental age of placentas. The mice were killed by cervical dislocation and the placentas were dissected at different time points. Isolated placentas were directly frozen by liquid nitrogen and stored at $-80^{\circ} \mathrm{C}$ until sectioning. The use of animals in this study was approved by the Ethics Committee of the Shenzhen PKUHKUST Medical Center.

\section{In situ hybridization}

The Plac1 probe sequences were cloned into the pGM-T plasmid (Tiangen, Beijing, China) with the T7 and SP6 promoters flanking both sides of the insertion site. The following forward and reverse primers were used to amplify a 333-bp Plac1 fragment (NM_019538): 5'-TTGAAGGCTAAGGGACCTGC-3', and 5'-TCTGTCGAGCACAGCACATT-3', respectively. The fragment was then cloned to the plasmid as a template to generate the probe. The probe was labeled using a digoxigenin (DIG) RNA labeling kit (SP6/T7) (Roche, Mannheim, Germany).

The mouse placenta was embedded by Tissue-Tek ${ }^{\circledR}$ OCT compound (Sakura, Tokyo, Japan). Briefly, 8- $\mu$ mthick sections were prepared from the frozen placental samples using a Leica CM 1950 cryostat (Wetzlar, Germany), and mounted on RNase-free glass slides. The sections were fixed by neutral paraformaldehyde (PFA; pH 7.4) for $1 \mathrm{~h}$ at $4^{\circ} \mathrm{C}$. ISH was performed as previously described [12]. Briefly, $2 \mu \mathrm{g} / \mathrm{mL}$ of DIG-labeled cRNA probe of Plac1 was used for hybridization at $60^{\circ} \mathrm{C}$ for $16 \mathrm{~h}$. The sections were then counterstained by nuclear fast red solution (N3020, Sigma). The sense probe was incubated with the serial sections and deemed as the negative control. The marker gene trophoblast-specific protein $\alpha$ (Tpbp) was used as the antisense probe to label the EPC or spongiotrophoblast layer [13]. Tissues from five different female mice were employed at each stage of pregnancy. 


\section{Cellular Physiology Cell Physiol Biochem 2017;43:2001-2009 and Biochemistry Published \begin{tabular}{l|l} 
DOI: 10.1159/000484154 & $\begin{array}{l}\text { C } 2017 \text { The Author(s). Published by S. Karger AG, Basel } \\
\text { www.karger.com/cpb }\end{array}$
\end{tabular}}

Gu et al.: Plac1 Expression Pattern and Function in Mouse Placentas

TS cell establishment and differentiation

TS cells were isolated from embryonic day (E) 3.5 mouse blastocysts according to the procedure described previously [14]. The established TS cell line was maintained in a proliferative state in mixed medium (70\% embryonic fibroblast conditioned medium and 30\% TS cell medium). The medium also contained $25 \mathrm{ng} / \mathrm{mL} \mathrm{FGF} 4$ and $1 \mathrm{mg} / \mathrm{mL}$ heparin. The TS cell medium containing CHIR99021 (Biovision, Milpitas, CA) and without bFGF, heparin, and embryonic fibroblast preconditioning was used for TS differentiation culture.

\section{Plac1 knockdown by specific shRNA lentivirus}

TS cells were previously infected with Plac1-specific shRNA-overexpressing lentivirus (pLL3.7) before the induced differentiation. A scrambled shRNA lentivirus was applied to TS cells as the control. The Plac1 shRNA sequence was 5'-TGCCCACCTTATGTCAATCAAA-3'. The lentiviral titer used was in line with the rule that each cell has the chance of being infected by ten lentivirus particles.

Real-time RT-PCR

Total RNA was extracted and purified from the TS differentiation group cultured for $0,2,4$, and 6 days. A corresponding TS control group was maintained according to the manufacturer's instructions of TRIzol ${ }^{\circledR}$ reagent (Invitrogen, Carlsbad, CA). The RNA concentration was examined by a NanoDrop 2000 UVVis spectrophotometer (Thermo Scientific, Wilmington, DE). RNA was reverse transcribed to cDNA using a PrimeScript" $\mathrm{RT}$ reagent kit (Takara, Dalian, China) with $1 \mu \mathrm{g}$ of total RNA per sample.

The relative transcript levels were quantified on a Real-time PCR System (ABI PRISM 7500 Real-time PCR System, Applied Biosystems, Foster City, CA) using a SYBR Premix Ex Taq kit (Takara, Dalian, China). Applied PCR was carried out according to the manufacturer's instructions of the SYBR Premix Ex Taq kit. Plac1 transcription levels were analyzed using the $\triangle \mathrm{Ct}$ method and normalized to the expression of Gapdh (NM_001289726) [15]. The primer pairs used for mouse Gapdh were 5'-GGTAAGGTCGGTGTGAACA-3' (forward) and 5'-CTCGCTCCTGGAAGATGGTG-3' (reverse). The primer pair for Plac1 (NM_019538) was 5'-TGCTCCACACGGAGAGAACA-3' (forward) and 5'-TGATTGGGAGGGCAACCAAG-3' (reverse). The marker genes used to indicate TS cell differentiation included Tpbpa, Pl-1, Esrrb, and Eomes, and the primers used for these genes were as described previously [16].

\section{Statistical analysis}

Data were illustrated as the means \pm SEM. Data were calculated by analysis of variance using Statistical Package for Social Science software (SPSS for Windows package release 10.0, SPSS, Inc.) followed by the $t$ test. Statistical significance was accepted as $P<0.05$. The experimental results involved in statistical calculations were performed in triplicate.

\section{Results}

The Tpbpa antisense probe was applied for ISH with serial frozen sections to identify the EPC of 8.5- and 9.5-dpc mouse placenta (Fig. 1, first row, left), or the spongiotrophoblast layer of a being formed placenta which observed at 10.5, 11.5 and $13.5 \mathrm{dpc}$ (Fig. 1, first row, right). As illustrated by the performance of the Plac1 antisense probe, Plac1 mRNA levels were abundant at the EPC of 8.5- and 9.5-dpc placentas (Fig. 1, second row, left). The complete spongiotrophoblast and labyrinth layers could be observed begin from $10.5 \mathrm{dpc}$, and the Plac1 expression levels remained high at the spongiotrophoblast layer but moderate at the labyrinth layer (Fig. 1, second row, middle). From 13.5 dpc, Plac1 expression in both these cell layers obviously decreased (Fig. 1, second row, right). In fact, Plac1 expression was not completely specific to the placenta. Plac1 expression in the fetus was also very high from 8.5 to $10.5 \mathrm{dpc}$, and decreased at $11.5 \mathrm{dpc}$ and thereafter. Plac1 expression level in the maternal decidua was weak (Fig. 1, second row). The sense probe incubated with serial frozen sections of mouse placenta at different developmental stages was employed as the negative control of ISH (Fig. 1, third row).

The fetomaternal interface consists of multiple components of cell subtypes and structures. At the fetomaternal interface, Plac1 exhibited trophoblast-specific expression, 


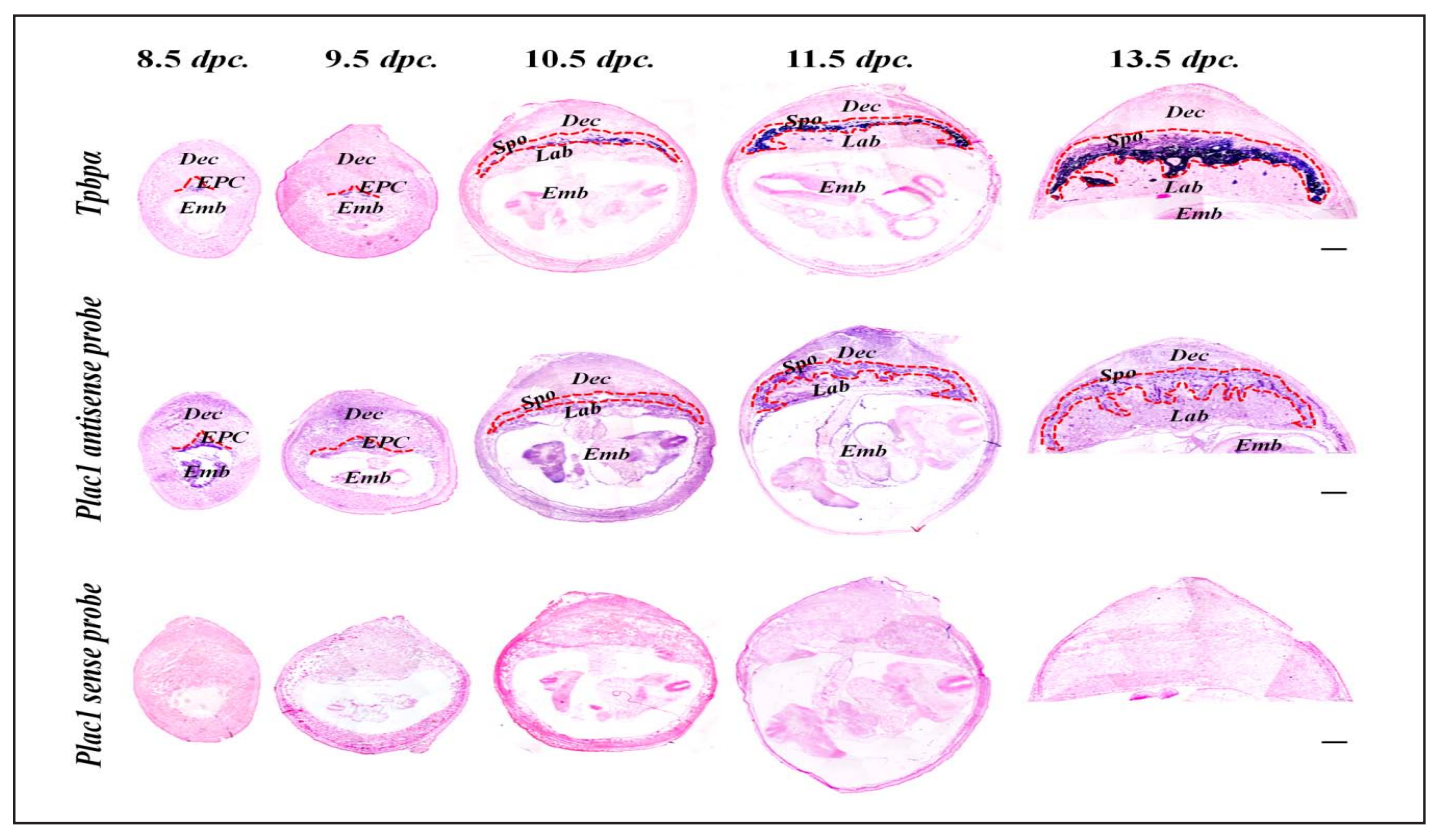

Fig. 1. An overview of Plac1 localization at the mouse fetomaternal interface at different stages of pregnancy. Frozen sections of placenta obtained at five different stages of placentation $(8.5,9.5,10.5,11.5$, and 13.5 $\mathrm{dpc}$ ) were employed. The Tpbp antisense probe was a marker to label the EPC ( 8.5 and $9.5 \mathrm{dpc}$ ) and the Spo layer of formed placenta (from $10.5 \mathrm{dpc}$ ). Plac1 mRNA was observed in the EPC and Emb in the 8.5- and 9.5-dpc specimens, or the Spo, Lab, and UC and Emb in the 10.5-dpc specimens. From 11.5 dpc, a positive signal could be observed at the maternal side, which appeared to be infiltrated by invading trophoblast cells. Plac1 expression level at the Emb side was obviously declined and restricted to some specific areas starting from 11.5 dpc. Furthermore, Plac1 expression levels were obviously decreased in Lab but remained high in Spo, the artery, and the Dec. EPC, ectoplacental cone. Dec, decidua. Emb, embryo. UC, umbilical cord. Spo, spongiotrophoblast, Lab, labyrinth. $\mathrm{n}=3$. Bar $=200 \mu \mathrm{m}$.

and was exclusively detected in the EPC of 8.5-dpc placentas, and it was expressed in both the spongiotrophoblast and labyrinth layers from 10.5 dpc (Fig. 2, line 1). However, Plac1 expression in both these layers began to decline from $13.5 \mathrm{dpc}$. When focusing on the maternal side, no obvious positive signals for the antisense probe was observed at any of the pregnancy stages studied (Fig. 2, line 2). A positive signal was observed at the decidual cells adjacent to the spongiotrophoblast layer at $11.5 \mathrm{dpc}$, which may have been produced by glycogen trophoblast cells or by giant cells that remodel the spiral artery (Fig. 2, 11.5 and $13.5 \mathrm{dpc}$, line 2).

Trophoblast giant cells and glycogen trophoblast cells are the invasive trophoblast subpopulations at the mouse fetomaternal interface. All glycogen trophoblast cells and some trophoblast giant cells were positive for the Plac1 antisense probe. There are two major types of trophoblast giant cells: primary trophoblast giant cells, which arise at $6.5 \mathrm{dpc}$ and secondary trophoblast giant cells, which emerge at $8.5 \mathrm{dpc}$. As illustrated in Fig. 2 (line 3), Plac1 expression was absent in primary giant cells (black star) but strongly present in secondary giant cells (blue star).

Although Plac1 was termed a placenta-specific gene, we observed a strong positive signal of Plac1 at the chorioallantois (Fig. 2, line 4) and at the umbilical cord (Fig. 1). Furthermore, Plac1 expression levels in the chorioallantois and umbilical cord increased from $8.5 \mathrm{dpc}$, peaked at $10.5 \mathrm{dpc}$ in the chorioallantois and at $11.5 \mathrm{dpc}$ in the umbilical cord, and decreased at $13.5 \mathrm{dpc}$. Similar expression patterns for Plac1 were also observed in the fetus, with the peak expression in the fetus ranging from 8.5-10.5 dpc, with an obvious decline from 11.5 dpc observed together with restricted localization (Fig. 1, and line 5 of Fig. 2). 


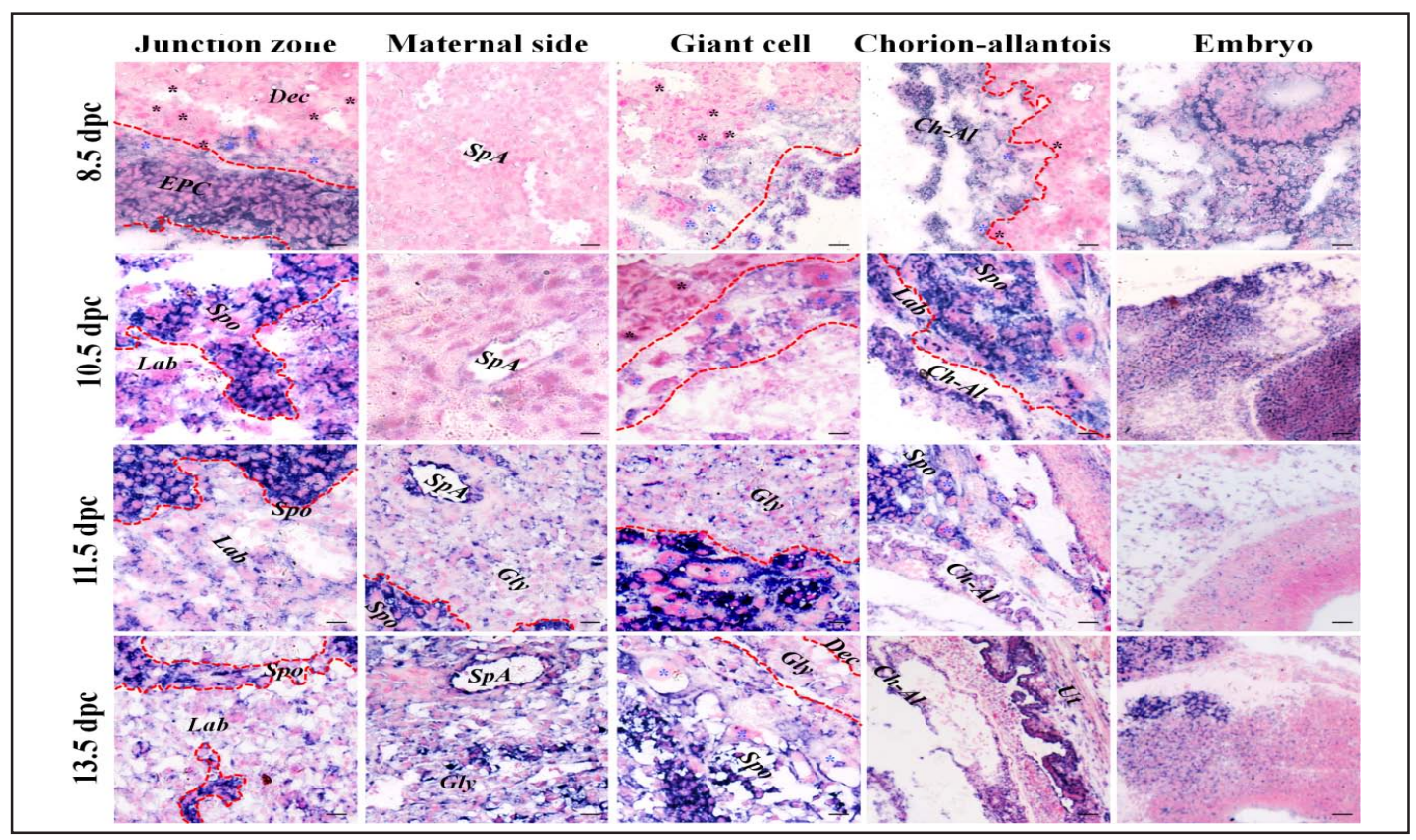

Fig. 2. The exact spatiotemporal expression pattern of Plac1 at the mouse fetomaternal interface. The signal produced by the Plac1 antisense probe incubation was mainly observed at the Spa and Lab as well as at the trophoblast component of the fetomaternal junction zone (first panel). Plac1 expression was the highest at the Spo of 10.5-dpc and 11.5-dpc specimens, respectively. The Plac1 mRNA level obviously declined at Spo and Lab from $13.5 \mathrm{dpc}$ onwards. The decidua and SpA located in the decidua were both negative for the Plac1 antisense probe (at 8.5 and $10.5 \mathrm{dpc}$, included in the second panel). However, Plac1 mRNA was observed in the decidua after $10.5 \mathrm{dpc}$ because of the invasion of trophoblast giant cells (in the SpA wall) and glycogen trophoblast cells adjacent to the Spo $(11.5,13.5$, and $10.5 \mathrm{dpc}$ sections included in the second panel). Plac1 expression exclusively detected in trophoblast components, except primary giant cells (black star indicates primary giant cells, and blue star indicates secondary giant cells). A strong positive signal for Plac1 mRNA was also observed in the chorioallantois (third panel). Strong Plac1 expression was detected in the embryo at an early stage of pregnancy (8.5- and 10.5-dpc sections; fifth panel), and the expression of Plac1 obviously declined from $11.5 \mathrm{dpc}$. Meanwhile Plac1 expression showed an obvious reduction and was restricted to specific areas of the embryo (fifth panel). EPC, ectoplacental cone. Dec, decidua. Emb, embryo. UC, umbilical cord. Spo, spongiotrophoblast. Gly, Glycogen trophoblast. Lab, labyrinth. Ut, uterus. Ch-Al, chorioallantois. SpA, spiral artery. $\mathrm{n}=3$. Bar $=100 \mu \mathrm{m}$.

An in vitro TS cells differentiation model was employed to indicate the possible role of Plac1 in trophoblast differentiation. The differentiation status of TS cells and control cells were indicated by transcriptional changes in the following marker genes: Tpbp, Pl-1, Esrrb, and Eomes (Fig. 3a-d). Plac1 transcription was absent in TS cells, but it was dramatically elevated along with the TS differentiation process (Fig. 3e). After 6 days of differentiation, the Plac1 level showed an increase of more than 500-fold $(P<0.01$; Fig. 3e).

Here, an in vitro TS cell differentiation model with lentiviral Plac1 knockdown was employed to discriminate the exact role of Plac1 in TS differentiation. The changes in the transcriptional levels of marker genes relative to the control were used to determine the differentiation level of TS. The corresponding changes in gene expression were determined by real-time RT-PCR. Similar to the results in Fig. 3, Eomes and Esrrb expression levels significantly decreased in the negative control lentivirus treatment with progression of TS differentiation (Fig. 4a and b). In contrast, although Eomes and Esrrb expression in the Plac1 knockdown group also significantly decreased after six days of differentiation, Eomes and Esrrb expression levels declined to a lesser extent relative to the control. The Eomes and Esrrb mRNA levels decreased by $100 \times$ and $3 \times$ the corresponding levels in the negative 
Fig. 3. Quantitative RT-PCR analysis of changes in Plac1 expression during the differentiation process of TS cells in vitro. TS differentiation was indicated by the downregulation of Eomes (a) and Esrrb (b) and the upregulation of Pl-I (c) and Tpbpa (d). Plac1 mRNA level increased in a timedependent manner and showed an increase of more than 500fold after TS cells underwent differentiation for 6 days. TS, trophoblast stem cells. $\mathrm{n}$ $=3 .{ }^{*}, \mathrm{p}<0.05 .{ }^{* *}$, $\mathrm{p}<0.01$.
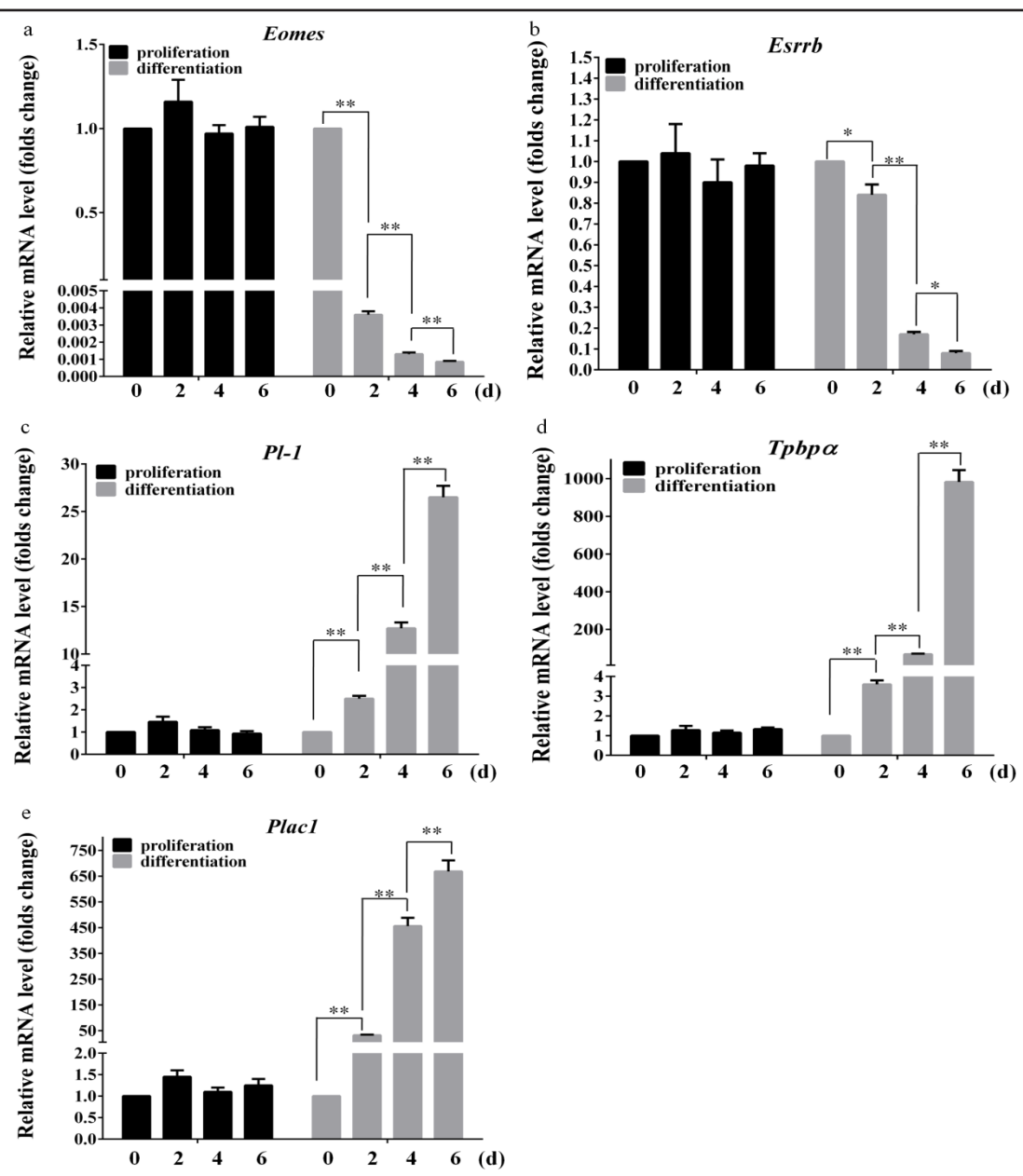

control respectively (Fig. 4c and d). The silencing efficiency for Plac1 shRNA lentivirus was approximately $80 \%$ (Fig. 4 e).

\section{Discussion}

Plac1 first attracted the attention of researchers due to a chromosomal deletion of 200$700 \mathrm{~kb}$ around the mouse Hprt locus, which is the location of the Plac1 gene, resulted in fetal growth retardation and early death [17]. Recently, Plac1 has been found to be highly expressed in the main trophoblast linage [18]. Plac1 mutation resulted in mouse placental defects, presented as expansion and excessive invasion of the labyrinth by the spongiotrophoblast layer [11]. Meanwhile, knockdown of Plac1 has been found to be associated with a lethal form of hydrocephalus $[11,19]$. The corresponding orthologous gene in humans, PLAC1, is expressed in a wide range of carcinoma and cancer cell lines and facilitates the invasion process [20]. Furthermore, several studies have reported the association between abnormal PLAC1 expression and adverse pregnancy outcomes [21-24]. This is the first study to identify the spatial and temporal expression pattern of Plac1 at the mouse fetomaternal interface.

As illustrated in our study, Plac1 is highly expressed in the EPC of $8.5 \mathrm{dpc}$ mice. Another study focusing on human trophoblasts have shown that PLAC1 is very important for the invasion of extravillous trophoblasts [12] and syncytialization [25]. Plac1 is abundantly expressed in mice, and is widely distributed in different trophoblast subpopulations. There 
Fig. 4. Plac1 knockdown significantly retarded the progression of TS cell differentiation. As indicated by the downregulation of the Eomes (a) and Esrrb (b) transcript levels and the upregulation of the Pl-I (c) and Tpbpa (d) transcript levels, knockdown of Plac1 expression (e) by lentivirus significant impaired the TS differentiation level although it could not completely block it,. TS, trophoblast stem cells. $\mathrm{n}=$ 3. ${ }^{*}, \mathrm{p}<0.05$. $^{* *}, \mathrm{p}<0.01$.
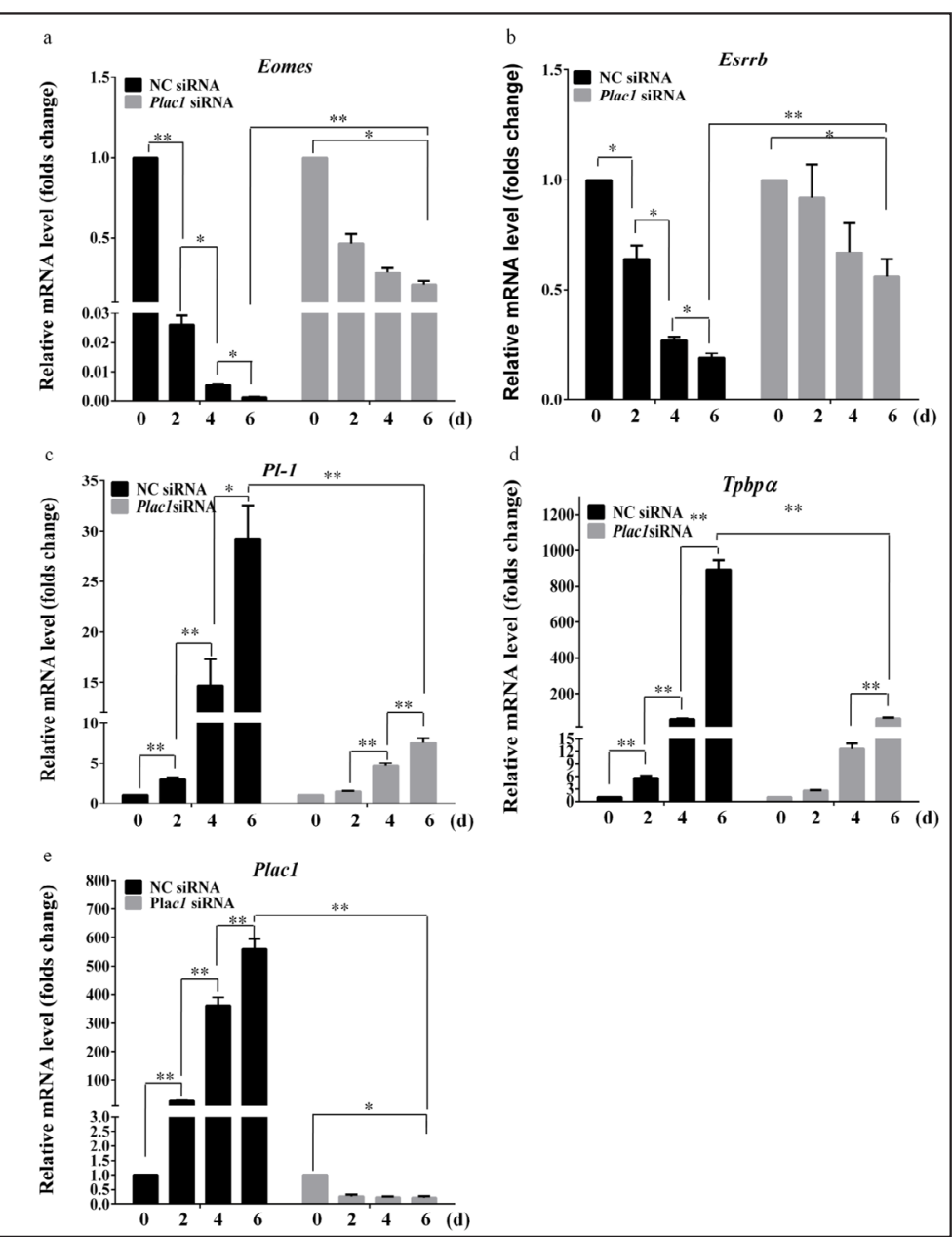

are three main invasive trophoblastic subpopulations at the fetomaternal interface of mice: primary trophoblast giant cells, which are observed from $6.5 \mathrm{dpc}$; secondary trophoblast giant cells, which emerge at $8.5 \mathrm{dpc}$; and glycogen trophoblasts, which can clearly be distinguished at $11.5 \mathrm{dpc}$. Plac1 is strongly expressed in secondary trophoblast giant cells and glycogen trophoblast cells but not in primary trophoblast giant cells.

Although Plac1 was initially suggested as a placenta-specific gene [18], it has been reported to be abundantly expressed in the fetus $[11,19]$, coinciding with the observation that Plac1 is not only essential for normal placentation but also for embryonic development. The results of our ISH also illustrated that the early embryo (8.5-10.5 dpc) had the highest and most widespread distribution of Plac1 expression. Then, the Plac1 expression level decreased and its distribution became restricted. Plac1 knockout in the placenta was found to be attributed to the abnormal structure of spongiotrophoblasts, and knockout of Plac1 in the embryo has been shown to induce hydrocephalus [11], which can be rescued by Plac1 overexpression [26]. Furthermore, the possible function of Plac1 in the chorioallantois, umbilical cord, and uterus during pregnancy deserves our attention because its abundance and lasting expression in the chorioallantois, umbilical cord, as well as the uterine epithelium from $13.5 \mathrm{dpc}$ was observed in our study.

Plac1, which is typically enriched in the placental cDNA library, is considered to play a significant role in pregnancy by participating in trophoblast differentiation. This was further reinforced by our study with the observation of significant transcription activation for Plac1 in the TS cell differentiation process. Although the Plac1 knockout mouse model was fertile, compromised fecundity has been previously observed [11]. Lentiviral knockdown of Plac1 


\section{Cellular Physiology Cell Physiol Biochem 2017;43:2001-2009

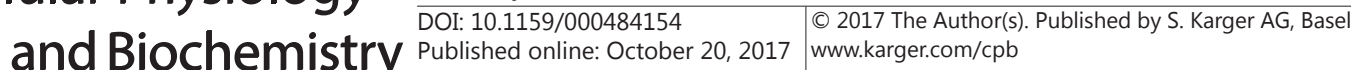

Gu et al.: Plac1 Expression Pattern and Function in Mouse Placentas

could significantly retard the progress of TS cell differentiation. In conclusion, our research suggested that Plac1 is very important for trophoblast differentiation and plays a unique role facilitating normal pregnancy in mice.

\section{Acknowledgements}

This study was supported by the grants from Basic Research projects of Shenzhen Science and Technology Innovation Committee, Shenzhen, Guangdong, China (JCYJ20160427185401364, JCYJ20170306161657672).

\section{Disclosure Statement}

The authors declared no potential conflicts of interest with respect to the research, authorship, and/or publication of this article.

\section{References}

1 Springer MS, Murphy WJ, Eizirik E, O’Brien SJ: Placental mammal diversification and the CretaceousTertiary boundary. Proc Natl Acad Sci U S A 2003;100:1056-1061.

-2 Wildman DE, Chen C, Erez O, Grossman LI, Goodman M, Romero R: Evolution of the mammalian placenta revealed by phylogenetic analysis. Proc Natl Acad Sci U S A 2006;103:3203-3208.

3 Rossant J, Cross JC: Placental development: lessons from mouse mutants. Nat Rev Genet 2001;2:538-548.

4 Rawn SM, Cross JC: The evolution, regulation, and function of placenta-specific genes. Ann Rev Cell Dev Biol 2008;24:159-181.

-5 Huang QT, Chen JH, Hang LL, Liu SS, Zhong M: Activation of PAR-1/NADPH oxidase/ROS signaling pathways is crucial for the thrombin-induced sFlt-1 production in extravillous trophoblasts: possible involvement in the pathogenesis of preeclampsia. Cell Physiol Biochem 2015;35:1654-1662.

-6 Fan M, Xu Y, Hong F, Gao X, Xin G, Hong H, Dong L, Zhao X: Rac1/beta-Catenin Signalling Pathway Contributes to Trophoblast Cell Invasion by Targeting Snail and MMP9. Cell Physiol Biochem 2016;38:1319-1332.

7 Maltepe E, Bakardjiev AI, Fisher SJ: The placenta: transcriptional, epigenetic, and physiological integration during development. J Clin Invest 2010;120:1016-1025.

8 Nelissen EC, van Montfoort AP, Dumoulin JC, Evers JL: Epigenetics and the placenta. Hum Reprod Update 2011;17:397-417.

-9 Li H, Han L, Yang Z, Huang W, Zhang X, Gu Y, Li Y, Liu X, Zhou L, Hu J, Yu M, Yang J, Li Y, Zheng Y, Guo J, Han J, Li L: Differential Proteomic Analysis of Syncytiotrophoblast Extracellular Vesicles from Early-Onset Severe Preeclampsia, using 8-Plex iTRAQ Labeling Coupled with 2D Nano LC-MS/MS. Cell Physiol Biochem 2015;36:1116-1130.

10 Liu Z, Ruan HJ, Gu PQ, Ding WY, Luo XH, Huang R, Zhao W, Gao LJ: The Roles of p38 MAPK and ERK1/2 in Coplanar Polychlorinated Biphenyls-Induced Apoptosis of Human Extravillous Cytotrophoblast-Derived Transformed Cells. Cell Physiol Biochem 2015;36:2418-2432.

-11 Jackman SM, Kong X, Fant ME: Plac1 (placenta-specific 1) is essential for normal placental and embryonic development. Mol Reprod Dev 2012;79:564-572.

$\checkmark 12$ Chang WL, Yang Q, Zhang H, Lin HY, Zhou Z, Lu X, Zhu C, Xue LQ, Wang H: Role of placenta-specific protein 1 in trophoblast invasion and migration. Reproduction 2014;148:343-352.

13 Gasperowicz M, Surmann-Schmitt C, Hamada Y, Otto F, Cross JC: The transcriptional co-repressor TLE3 regulates development of trophoblast giant cells lining maternal blood spaces in the mouse placenta. Dev Biol 2013;382:1-14.

14 Tanaka S, Kunath T, Hadjantonakis AK, Nagy A, Rossant J: Promotion of trophoblast stem cell proliferation by FGF4. Science 1998;282:2072-2075. 


\section{Cellular Physiology Cell Physiol Biochem 2017;43:2001-2009 \begin{tabular}{ll|l} 
DOI: 10.1159/000484154 2017 and Biochemistry Published online: October 20, 2017 & $\begin{array}{l}\text { O 2017 The Author(s). Published by S. Karger AG, Basel } \\
\text { www.karger.com/cpb }\end{array}$
\end{tabular}}

15 Schmittgen TD, Livak KJ: Analyzing real-time PCR data by the comparative C(T) method. Nat Protoc 2008;3:1101-1108.

16 Lu J, Zhang S, Nakano H, Simmons DG, Wang S, Kong S, Wang Q, Shen L, Tu Z, Wang W, Wang B, Wang H, Wang Y, van Es JH, Clevers H, Leone G, Cross JC, Wang H: A positive feedback loop involving Gcm1 and Fzd5 directs chorionic branching morphogenesis in the placenta. PLoS Biol 2013;11:e1001536.

17 Kushi A, Edamura K, Noguchi M, Akiyama K, Nishi Y, Sasai H: Generation of mutant mice with large chromosomal deletion by use of irradiated ES cells--analysis of large deletion around hprt locus of ES cell. Mamm Genome 1998;9:269-273.

18 Cocchia M, Huber R, Pantano S, Chen EY, Ma P, Forabosco A, Ko MS, Schlessinger D: PLAC1, an Xq26 gene with placenta-specific expression. Genomics 2000;68:305-312.

19 Kong X, Jackman SM, Fant ME: Plac1 (placenta-specific 1) is widely expressed during fetal development and is associated with a lethal form of hydrocephalus. Birth Defects Res A Clin Mol Teratol 2013;97:571-577.

-20 Koslowski M, Sahin U, Mitnacht-Kraus R, Seitz G, Huber C, Tureci O: A placenta-specific gene ectopically activated in many human cancers is essentially involved in malignant cell processes. Cancer Res 2007;67:9528-9534.

21 Purwosunu Y, Sekizawa A, Farina A, Wibowo N, Okazaki S, Nakamura M, Samura O, Fujito N, Okai T: Cellfree mRNA concentrations of CRH, PLAC1, and selectin-P are increased in the plasma of pregnant women with preeclampsia. Prenat Diagn 2007;27:772-777.

22 Kodama M, Miyoshi H, Fujito N, Samura O, Kudo Y: Plasma mRNA concentrations of placenta-specific 1 (PLAC1) and pregnancy associated plasma protein A (PAPP-A) are higher in early-onset than late-onset pre-eclampsia. J Obstet Gynaecol Res 2011;37:313-318.

-23 Matteo M, Greco P, Levi Setti PE, Morenghi E, De Rosario F, Massenzio F, Albani E, Totaro P, Liso A: Preliminary evidence for high anti-PLAC1 antibody levels in infertile patients with repeated unexplained implantation failure. Placenta 2013;34:335-339.

-24 Zanello M, Sekizawa A, Purwosunu Y, Curti A, Farina A: Circulating mRNA for the PLAC1 gene as a second trimester marker (14-18 weeks' gestation) in the screening for late preeclampsia. Fetal Diagn Ther 2014;36:196-201.

25 Chang WL, Wang H, Cui L, Peng NN, Fan X, Xue LQ, Yang Q: PLAC1 is involved in human trophoblast syncytialization. Reprod Biol 2016;16:218-224.

-26 Muto M, Fujihara Y, Tobita T, Kiyozumi D, Ikawa M: Lentiviral Vector-Mediated Complementation Restored Fetal Viability but Not Placental Hyperplasia in Plac1-Deficient Mice. Biol Reprod 2016;94:6. 